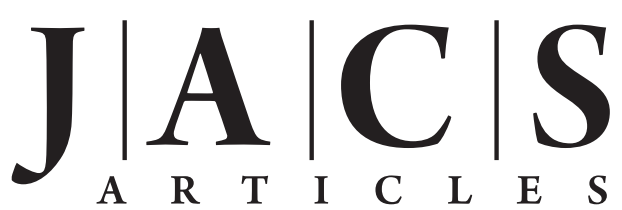

Published on Web 09/24/2010

\title{
Through-Bond Interactions in the Diradical Intermediates Formed in the Rearrangements of Bicyclo[n.m.0]alkatetraenes
}

\author{
Charity Flener Lovitt, ${ }^{\dagger}$ Hao Dong, ${ }^{\dagger}$ David A. Hrovat, ${ }^{\dagger}$ Rolf Gleiter, ${ }^{\ddagger}$ and \\ Weston Thatcher Borden*,† \\ Department of Chemistry and Center for Advanced Scientific Computing and Modeling, \\ University of North Texas, 1155 Union Circle No. 305070, Denton, Texas 76203-5017, and \\ Organisch-Chemisches Institut der Universität Heidelberg Im Neuenheimer, Feld 270, \\ D-69120 Heidelberg, Germany \\ Received July 16, 2010; E-mail: borden@unt.edu
}

\begin{abstract}
The intermediates and transition structures in the degenerate thermal rearrangements of bicyclo[4.4.0]deca-2,4,7,9-tetraene (1c), bicyclo[5.5.0]dodeca-2,4,8,10-tetraene(11b), and bicyclo[5.4.0]undeca2,4,8,10-tetraene (14) have been located by (U)B3LYP/6-31G(d) calculations. The singlet-triplet energy differences $\left(\Delta E_{S T}\right)$ in the diradical intermediates (tricyclo[4.4.0.0 2,7 deca-3,8-dien-5,10-diyl (2c), tricyclo[5.5.0.0 $0^{5,11}$ ]dodeca-2,8-dien-4,10-diyl (12b), and tricyclo[5.4.0.0 $\left.0^{5,11}\right]$ undeca-2,8-dien-4,10-diyl (15)) have been computed, using both UB3LYP and (6/6)CASPT2 calculations. $\Delta E_{\mathrm{ST}}$ in $2 \mathrm{c}$, in which a fourmembered ring is anti-bridged by two allylic radicals, is computed to be larger by a factor of 5 than $\Delta E_{\mathrm{ST}}$ in 15, in which the anti-bridged ring is five-membered, and by a factor of 10 than that in $12 \mathrm{~b}$, in which the anti-bridged ring is six-membered. The reasons for the much larger interaction between two allylic radicals through the bonds of the four-membered ring in $\mathbf{2 c}$ than through the bonds of the fivemembered ring in $\mathbf{1 5}$ or the six-membered ring in 12b are discussed, and the consequences of the large, through-bond stabilization of the singlet state of $\mathbf{2 c}$ are described.
\end{abstract}

\section{Introduction}

Thermal isomerization of the 9,10-disubstituted cis-9,10dihydronaphthalenes $\mathbf{1 a}$ and $\mathbf{1 b}$ affords predominantly the 1,5and 1,9 -disubstituted products $\mathbf{3 a} / \mathbf{3} \mathbf{b}$ and $\mathbf{4 a} / \mathbf{4 b}$, respectively. ${ }^{1}$

In order to rationalize these results, Paquette and Carmody proposed that two reaction channels were involved: ring-opening to all-cis-cyclodecapentaene, followed by ring closure, to afford 3 and 1,5-shift of a $\mathrm{C}-\mathrm{C} \sigma$ bond to give 4 .

This proposal was subsequently challenged by Alder and Grimme, ${ }^{2}$ who suggested that tricyclo[4.4.0.0 $\left.0^{2,7}\right]$ deca-3,8-dien5,10-diyl (2) is a common intermediate in the formation of both 3 and 4, as illustrated in Figure 1. On the basis of strong throughbond interactions between the nonbonding orbitals of the two perpendicular allylic radicals in $\mathbf{2}$, via the Walsh orbitals of the central four-membered ring, a singlet ground state had already been predicted for this diradical. ${ }^{3}$ The strong through-bond stabilization predicted for singlet $\mathbf{2}$ makes this species attractive as a possible intermediate in the rearrangement of $\mathbf{1}$ to $\mathbf{3}$ and $\mathbf{4}$.

The proposal of $\mathbf{2}$ as an intermediate in the rearrangement of $\mathbf{1}$ to $\mathbf{3}$ and $\mathbf{4}$ was supported by studies of three octamethyl9,10-dihydronaphthalene derivatives $(\mathbf{5}-\mathbf{7})$ by Maier et al. ${ }^{4}$ All three isomers interconvert at room temperature to afford a 1:1:1 mixture of 5-7 (Figure 2).

\footnotetext{
† University of North Texas.

Heidelberg University.

(1) Paquette, L. A.; Carmody, M. J. J. Am. Chem. Soc. 1975, 97, 5841.

(2) Alder, R. W.; Grimme, W. Tetrahedron 1981, 37, 1809.

(3) Bischof, P.; Gleiter, R.; Haider, R. J. Am. Chem. Soc. 1978, 100, 1036.

(4) Maier, G.; Wiegand, N. H.; Baum, S.; Wüllner, R.; Mayer, W.; Boese, R. Chem. Ber. 1989, 122, 767.
}

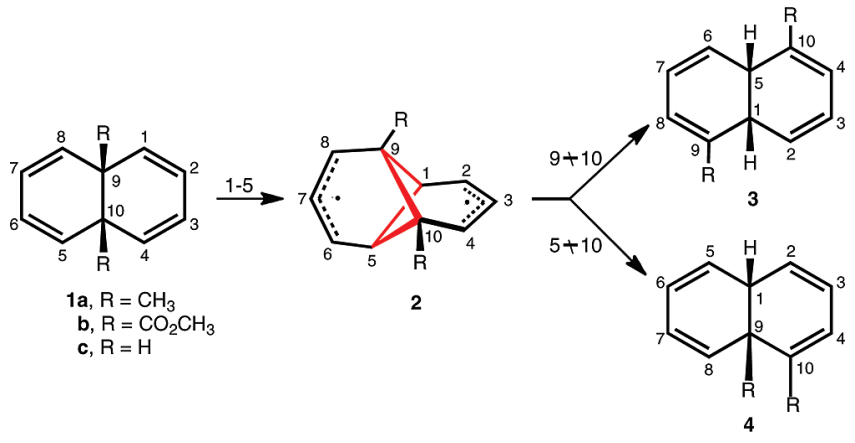

Figure 1. Thermolysis of $\mathbf{1 a}$ and $\mathbf{1 b}$ to yield $\mathbf{3 a} / \mathbf{4 a}$ and $\mathbf{3 b} / \mathbf{4 b}$, respectively. The numbering of the carbons in $\mathbf{2 - 4}$ indicates their relationship to the carbons in $\mathbf{1}$, and the numbers over the arrows indicate the carbons between which bonds are made or broken. The $\mathrm{C}-\mathrm{C}$ bonds of the doubly bridged four-membered ring in $\mathbf{2}$ are shown in red.

In order to rationalize the fact that when $\mathbf{5 , 6}$, or $\mathbf{7}$ is dissolved in $\mathrm{CHCl}_{3}$ or benzene, only two out of the 15 possible constitutional isomers of the reactant are formed, Maier et al. scrutinized all five of the possible mechanisms that had been discussed in the literature. ${ }^{1,2}$ Maier concluded that only the formation of intermediate 9 (Figure 2) provides a consistent explanation of his results. ${ }^{4}$ Interestingly, other isomers of 5-7, such as might have been formed from diradicals $\mathbf{8}$ or $\mathbf{1 0}$, were not detected under the reaction conditions.

Related to the work on the rearrangements of 9,10-dihydronaphthalenes, described above, is the research reported by 


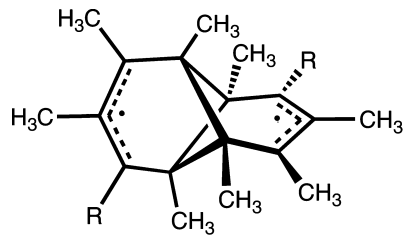

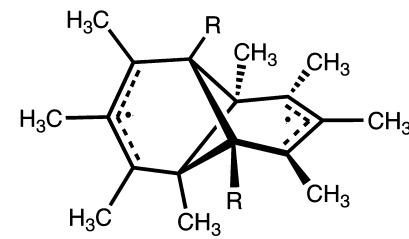

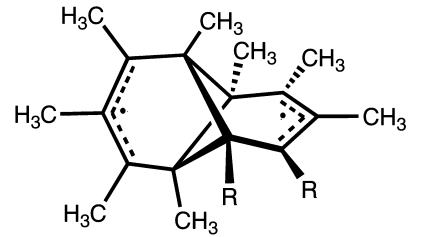<smiles>C=COCC(=C)C(C)(C)C</smiles><smiles>[2H]C1=C(C)C(C)=C(C)C2(C)C(C)=C(C)C(C)=C(C)C12C</smiles><smiles>C=C=C</smiles><smiles>C=C=CC</smiles>

10<smiles>CC1=C(C)C2(C)C(C)=C(C)C(C)C2(C)C(C)=C1C</smiles><smiles>[2H]C1=C(C)C(C)=C(C)[C@]2(C)C(C)=C(C)C(C)=C(C)[C@]12C</smiles>

Figure 2. Thermal equilibrium among $\mathbf{5 , 6}$, and $\mathbf{7}$ at room temperature via intermediate $\mathbf{9}$. The observation of only 5-7 argues against the formation of significant amounts of intermediates $\mathbf{8}$ and $\mathbf{1 0}$ under the reaction conditions.

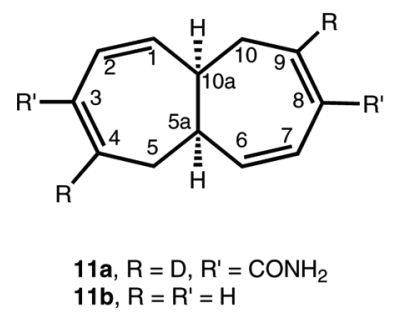

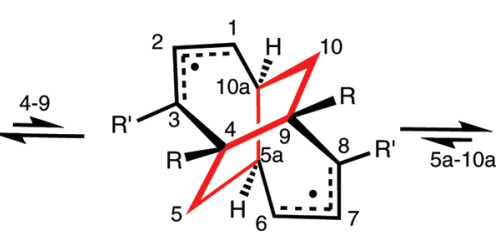

12

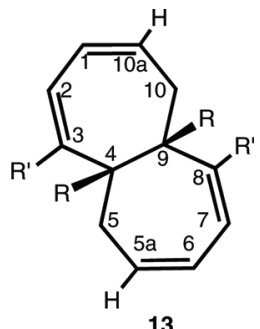

13

Figure 3. Equilibration of $\mathbf{1 1}$ and $\mathbf{1 3}$ via diradical 12. The numbering of the carbons in $\mathbf{1 2}$ and $\mathbf{1 3}$ indicates their relationship to the carbons in $\mathbf{1 1}$. The $\mathrm{C}-\mathrm{C}$ bonds of the doubly bridged six-membered ring in $\mathbf{1 2}$ are shown in red.

Hafner et al. ${ }^{5}$ on the thermal behavior of 5,5a,10,10a-tetrahydroheptalene derivatives, such as 11a and 13a. The thermal equilibration at $25^{\circ} \mathrm{C}$ of 11a and 13a, shown in Figure 3, was explained by assuming that the tricyclic diradical 12a lies on the reaction pathway. This postulate accounts for the labeling patterns in 11a and 13a and for the large, negative entropy of activation $\left(\Delta S^{\ddagger}=-13 \mathrm{eu}\right)$ for their equilibration.

Like the rearrangement of $\mathbf{1}$ to $\mathbf{3}$ and $\mathbf{4}$ and the equilibration of 5-7, the equilibration of $\mathbf{1 1}$ and $\mathbf{1 3}$ can be viewed as the bis-vinylog of a Cope rearrangement. However, the results of DFT calculations, published by Houk and co-workers, ${ }^{6}$ found that the [5,5]sigmatropic shift that equilibrates $\mathbf{1 1}$ and $\mathbf{1 3}$ is not concerted but, instead, involves formation of diradical $\mathbf{1 2}$ as an energy minimum on the singlet potential energy surface for this reaction.

Compound $\mathbf{1 1}$ is a bis-homologue of $\mathbf{1}$. Therefore, diradicals $\mathbf{2}$ and $\mathbf{1 2}$ differ by the size of the ring that is bridged by the two allylic radicals, a four-membered ring in 2 and a sixmembered ring in 12. As already noted, the large orbital energy differences (calculated by EH and by MINDO/3) between the two symmetry combinations of the allylic nonbonding orbitals in $\mathbf{2}$ were attributed to through-bond interactions between these orbitals, involving the bonds of the puckered four-membered ring. ${ }^{3}$ An obvious question is, therefore, whether the bonds of the six-membered ring in $\mathbf{1 2}$ function similarly and create a large

(5) Hafner, K.; Lindner, H. J.; Weimel, L.; Meinhardt, K.-P.; Zink, T. Pure Appl. Chem. 1993, 65, 17.

(6) Beno, B. R.; Fennen, J.; Houk, K. N.; Lindner, H. J.; Hafner, K. J. Am. Chem. Soc. 1998, 120, 10490.

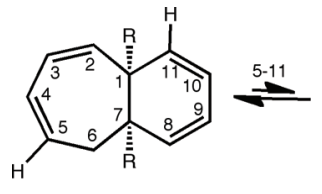

$14, R=H$

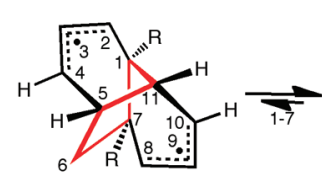

15

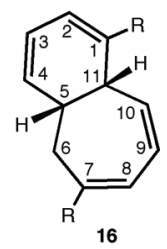

16
Figure 4. Possible equilibration of $\mathbf{1 4}$ and $\mathbf{1 6}$ via diradical 15. The numbering of the carbons in $\mathbf{1 5}$ and $\mathbf{1 6}$ indicates their relationship to the carbons in $\mathbf{1 4}$. The $\mathrm{C}-\mathrm{C}$ bonds of the doubly bridged five-membered ring in $\mathbf{1 5}$ are shown in red.

energy difference between the two symmetry combinations of the allylic nonbonding orbitals in this diradical.

The same question can be asked about the bonds of the five-membered ring in diradical 15. It might serve as an intermediate in the as-yet unknown equilibration of bicyclo[5.4.0] undeca-2,4,8,10-tetraenes $\mathbf{1 4}$ and $\mathbf{1 6}$. This reaction is shown in Figure 4.

How does the size of the bridged ring affect the ability of its bonds to mediate the interaction between the allylic nonbonding orbitals in diradicals 2, 12, and 15? Does the strength of the through-bond interactions in each of these diradicals depend on the energies of the ring orbitals with which the allylic nonbonding orbitals interact, on the number of ring bonds that interact with the terminal atoms of both allylic radicals (four bonds in 2, two in 12, and three in 15), or on some other factor(s)? Why are barriers to the rearrangements of $\mathbf{1 a}$ and $\mathbf{1 b}$ $\left(\Delta H^{\ddagger}=25 \mathrm{kcal} / \mathrm{mol} \text { for both }\right)^{1}$ so low, when both of these rearrangements require formation of a strained four-membered 
ring in a putative diradical intermediate? Are stabilizing throughbond interactions in diradicals $\mathbf{2} \mathbf{a}$ and $\mathbf{2} \mathbf{b}$ and in the transition structures leading to these diradicals responsible for the low barriers to the rearrangements of $\mathbf{1 a}$ and $\mathbf{1 b}$ ?

In order to address these questions, we have carried out both $a b$ initio and unrestricted (U)DFT calculations of the energy difference $\left(\Delta E_{\mathrm{ST}}\right)$ between the lowest singlet and triplet states of diradicals $\mathbf{2 c}, \mathbf{1 2 b}$, and $\mathbf{1 5}$. In such a closely related series of diradicals, $\Delta E_{\mathrm{ST}}$ should provide a good and, at least in principle, an experimentally measurable indicator of the extent to which the degeneracy between the two symmetry combinations of nonbonding allylic orbitals is lifted by mixing with the bonds of the bridged rings. In this paper, we report the results of our calculations and our conclusions about the interactions between the nonbonding allylic orbitals and the bonds of the bridged rings in diradicals $\mathbf{2 c}, \mathbf{1 2 b}$, and $\mathbf{1 5}$.

\section{Computational Methodology}

We have previously found ${ }^{7}$ that complete active-space (CAS)SCF calculations, ${ }^{8}$ followed by inclusion of the effects of dynamic electron correlation, ${ }^{9}$ afford singlet-triplet splittings that are generally in very good agreement with the experimentally measured values. For these calculations, the $6-31 \mathrm{G}(\mathrm{d})$ basis set ${ }^{10}$ has usually proven adequate.

Therefore, we performed (6/6)CASSCF/6-31G(d) calculations on the lowest singlet and triplet states of $\mathbf{2 c}, \mathbf{1 2 b}$, and $\mathbf{1 5}$. These calculations correlated the six electrons in the two bonding and two nonbonding allylic $\pi$ MOs in each diradical by using all the possible configurations that can be formed by excitations of electrons into the two antibonding $\pi^{*}$ MOs. Dynamic electron correlation was included through the use of multireference, secondorder perturbation theory (CASPT2). ${ }^{11}$ The CASSCF and CASPT2 calculations were carried out with MOLCAS. ${ }^{12}$

$\Delta E_{\mathrm{ST}}$ values are one way of establishing the extent to which the degeneracy between the two symmetry combinations of the allylic nonbonding MOs is lifted by through-bond interactions in $\mathbf{2 c}, \mathbf{1 2 b}$, and 15. Another indicator is the natural-orbital occupation numbers (NOONs) for these two orbitals in the CASSCF wave function for the lowest singlet state. Degenerate MOs would both have NOONs $=1.0$. If the degeneracy were very strongly lifted, then the NOON for the HOMO in the CASSCF wave function would approach 2.0 and the NOON for the LUMO would approach 0.0.

As an alternative to carrying out CASSCF and CASPT2 calculations on a diradical, a less rigorous but computationally easier way to calculate $\Delta E_{\mathrm{ST}}$ is to perform unrestricted (U)DFT calculations. In UDFT calculations a singlet is represented by configurations in which one electron is placed in each of two nonorthogonal MOs with the electron spins antiparallel. However, such a "singlet" wave function is not really a pure singlet but is, instead, a mixture of pure singlet and triplet wave functions. ${ }^{13}$ To the extent that the nonbonding electrons in such a "singlet" wave function both occupy the lower energy of the two symmetry combinations of the localized nonbonding (NB)MOs, the $\left\langle S^{2}\right\rangle$ value of the wave function will approach the value of $S^{2}=0$ for a pure singlet. At the other extreme,

(7) Review: Borden, W. T. In Magnetic Properties of Organic Materials; Lahti, P. M., Ed.; Marcel Dekker: New York, 1999; pp 61-102.

(8) Review: Roos, B. O. Adv. Chem. Phys. 1987, 69, 399.

(9) Borden, W. T.; Davidson, E. R. Acc. Chem. Res. 1996, $29,87$.

(10) Hariharan, P. C.; Pople, J. A. Theor. Chim. Acta. 1973, 28, 213.

(11) Anderson, K.; Malmqvist, P. A.; Roos., B. O. J. Chem. Phys. 1992, 96, 1218. Andersson, K.; Malmqvist, P. A.; Roos., B. O.; Sadlej, A. J.; Wolinski, K. J. Chem. Phys. 1990, 94, 5483.

(12) MOLCAS, version 6.4: Karlstrøm, G.; Lindh, R.; Malmqvist, P.-Å.; Roos, B. O.; Ryde, U.; Veryazov, V.; Widmark, P.-O.; Cossi, M.; Schimmelpfennig, B.; Neogrady, P.; Seijo, L. Comput. Mater. Sci. 2003, 28, 222.

(13) See, for example: Bally, T.; Borden, W. T.; Lipkowitz, K. B.; Doyd, D. B. Rev. Comput. Chem. 1999, 13, 1-97. to the extent that the two electrons in the "singlet" occupy different orbitals, which are localized in different regions of space from each other, the $\left\langle S^{2}\right\rangle$ value for the "singlet" wave function will be $\left\langle S^{2}\right\rangle \approx$ 1.0 , the average of $S^{2}=0$ for a pure singlet and $S^{2}=2.00$ for a pure triplet.

Thus, not only the values of $\Delta E_{\mathrm{ST}}$ and the CASSCF NOONs but also the value of $\left\langle S^{2}\right\rangle$ for a UDFT "singlet" wave function provide an indication of the extent to which a molecule has a closedshell electronic structure, with a large energy gap between the HOMO and LUMO, or is a diradical, with two nearly degenerate MOs. In the former case, $\Delta E_{\mathrm{ST}} \gg 0$, the ratio of the NOONs $>1$, and $S^{2}=0$ for the lowest "singlet". In the latter case, $\Delta E_{\mathrm{ST}} \approx 0$, the ratio of the NOONs $\approx 1$, and $\left\langle S^{2}\right\rangle \approx 1.0$ for the lowest "singlet".

All of these indicators provide qualitative information about the relative energies of the two partially occupied MOs in a diradical. It is possible to calculate the actual energies of these orbitals by computing the energy necessary to remove an electron from each of these MOs in the triplet state of a diradical. In the triplet, one electron occupies each of these MOs, so the difference between the ionization energies (IEs) of these two electrons is just equal to the energy difference between the two states of the radical cation in which one electron occupies one of these MOs, and the other MO is left empty.

We performed UDFT calculations with the B3LYP functional ${ }^{14}$ and the $6-31 \mathrm{G}(\mathrm{d})$ basis set. ${ }^{9}$ UB3LYP geometries of the lowest "singlet" and triplet states of $\mathbf{2 c}, \mathbf{1 2 b}$, and $\mathbf{1 5}$ were optimized using the Gaussian03 package of programs, ${ }^{15}$ and (6/6)CASSCF and CASPT2 single-point calculations were carried out at these optimized geometries. (5/6)CASSCF and CASPT2 single-point calculations were also performed at the optimized UB3LYP triplet geometries on the two radical cations, formed by removing one of the two unpaired electrons in the triplet state.

The geometries of the closed-shell reactant molecules $(\mathbf{1 c}, \mathbf{1 1 b}$, and 14 in, respectively, Figures 1, 3, and 4) were optimized with B3LYP/6-31G(d) calculations. The transition structures connecting $\mathbf{1 c}$ to $\mathbf{2 c}, \mathbf{1 1 b}$ to $\mathbf{1 2 b}$, and $\mathbf{1 4}$ to $\mathbf{1 5}$ were located with UB3LYP/ 6-31G(d) calculations. The (U)B3LYP/6-31G(d) geometries of the closed-shell reactants, the diradical intermediates, and the transition structures that connect them are available in the Supporting Information for this manuscript.

\section{Results and Discussion}

Our calculations found that like diradical $\mathbf{1 2 b},{ }^{6}$ singlet diradicals 2c and $\mathbf{1 5}$ are minima on the UB3LYP/6-31G(d) potential energy surface. The UB3LYP barriers to ring-opening of $2 \mathrm{c}$ to $1 \mathrm{c}$ (or $\mathbf{3 c}$ ), 15 to $\mathbf{1 4}$ (or 16), and $\mathbf{1 2 b}$ to $\mathbf{1 1 b}$ (or 13b) are, respectively, $\Delta H^{\ddagger}=2.1,5.2$, and $5.2 \mathrm{kcal} / \mathrm{mol}$. Passage over the transition structure is computed to have $\Delta H^{\ddagger}=27.7$ $\mathrm{kcal} / \mathrm{mol}$ for $\mathbf{1 c} \rightarrow \mathbf{2 c}, 24.5 \mathrm{kcal} / \mathrm{mol}$ for $\mathbf{1 4} \rightarrow \mathbf{1 5}$, and 29.1 $\mathrm{kcal} / \mathrm{mol}$ for $\mathbf{1 1 b} \rightarrow \mathbf{1 2 b}{ }^{6}$ The calculated activation enthalpy and entropy $\left(\Delta S^{\ddagger}=-5.2 \mathrm{eu}\right)$ for $\mathbf{1 c} \rightarrow \mathbf{2 c}$ are both in reasonable agreement with the experimental values of $\Delta H^{\ddagger}=25 \mathrm{kcal} / \mathrm{mol}$ and $\Delta S^{\ddagger}=-5.0$ eu reported by Paquette and Carmody ${ }^{1}$ for the rearrangements of $\mathbf{1 a}$ and $\mathbf{1} \mathbf{b}$, shown in Figure 1.

Singlet diradicals $\mathbf{2 c}$ and $\mathbf{1 2 b}$ are computed to be higher in enthalpy than 1c and 11b by, respectively, 25.6 and $23.9 \mathrm{kcal} /$ mol. The very similar enthalpies of tricyclic diradicals $\mathbf{2 c}$ and $\mathbf{1 2 b}$, relative to bicyclic tetraenes $\mathbf{1 c}$ and $\mathbf{1 1 b}$, are quite surprising. As noted in the introduction, formation of $\mathbf{2 c}$ from 1c requires the creation of a highly strained four-membered ring,

(14) Becke, A. D. J. Chem. Phys. 1993, 98, 5648. (b) Lee, C.; Yang, W.; Parr, R. G. Phys Rev. B 1988, 37, 78. (c) Miehlich, B.; Savin, A.; Stoll, H.; Preuss, H. Chem. Phys. Lett. 1989, 157, 200.

(15) Frisch, M. J.; et al. Gaussian 03, revision E.01; Gaussian, Inc.: Wallingford, CT, 2004. The full reference is given in the Supporting Information. 
Table 1. UB3LYP and (6/6)CASPT2/6-31G(d) Values of the Singlet-Triplet Energy Differences $\left(\Delta E_{\mathrm{ST}}\right)$ in Diradicals 2c, 15, and $\mathbf{1 2 b}$ and of the Ionization Energies (IEs) ${ }^{a}$ of the Two Singly-Occupied MOs in the Triplet States $(\mathrm{kcal} / \mathrm{mol})$. The $\left\langle S^{2}\right\rangle$ Values in the "Singlet" UHF Calculations and the Natural Orbital Occupancy Numbers (NOONs) of the HOMO and LUMO in the (6/6)CASSCF Singlet Wave Functions Are Also Given

\begin{tabular}{cccrccc}
\hline diradical & $\begin{array}{c}\Delta E_{\mathrm{ST}} \\
(\mathrm{UB})^{b}\end{array}$ & $\left\langle S^{2}\right\rangle^{c}$ & \multicolumn{1}{c}{$\begin{array}{c}\Delta E_{\mathrm{ST}} \\
(\mathrm{PT} 2)^{d}\end{array}$} & \multicolumn{1}{c}{ NOONs $^{e}$} & $\begin{array}{c}\text { IES } \\
(\mathrm{UB} 3)^{f}\end{array}$ & $\begin{array}{c}\text { IES } \\
(\mathrm{PT} 2)^{g}\end{array}$ \\
\hline $\mathbf{2 c}$ & 21.4 & 0.0 & 25.5 & $1.62,0.38$ & $130.3,173.4$ & $124.9,173.7$ \\
$\mathbf{1 5}$ & 3.7 & 0.85 & 5.9 & $1.31,0.69$ & $139.0,156.8$ & $135.3,155.5$ \\
$\mathbf{1 2 b}$ & 1.6 & 0.96 & 2.4 & $1.20,0.80$ & $140.5,154.2$ & $137.2,153.2$
\end{tabular}

${ }^{a}$ The negative of the IE of an electron in an $\mathrm{MO}$ can be taken as the energy of that MO. ${ }^{b}$ UB3LYP values of $\Delta E_{\mathrm{ST}} .{ }^{c}$ Values of $\left\langle S^{2}\right\rangle$ in the "singlet" UB3LYP wave functions. ${ }^{d}(6 / 6)$ CASPT2 values of $\Delta E_{\mathrm{ST}}$. ${ }^{e}$ Natural orbital occupation numbers in the (6/6)CASSCF wave functions for the singlets. ${ }^{f}$ UB3LYP ionization energies of the two singly occupied MOs in the triplet. ${ }^{g}$ CASPT2 ionization energies of the two singly occupied MOs in the triplet.

whereas a presumably much less strained six-membered ring is created in the formation of $\mathbf{1 2 b}$ from $\mathbf{1 1 b}$.

Also worthy of note is that $\mathbf{1 5}$ can undergo ring-opening to bicyclo[4.4.1] undeca-2,4,7,9-tetraene (17), as well as to $\mathbf{1 4}$ and 16. However, the bond between C-9 and C-10 in $\mathbf{1 5}$ overlaps poorly with the two sets of allylic $\pi$ orbitals. Therefore, the UB3LYP barrier to the ring-opening reaction of $\mathbf{1 5}$ to $\mathbf{1 7}$ is computed to be $5.1 \mathrm{kcal} / \mathrm{mol}$ higher than that for ring-opening of 15 to 14 or 16. Consequently, our calculations predict that an experimental study would find the degenerate rearrangement of $\mathbf{1 4}$ to $\mathbf{1 6}$ to be much faster than the formation of $\mathbf{1 7}$.

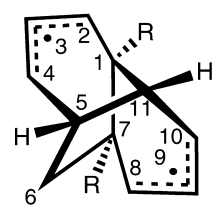

15

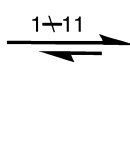

The results of our CASPT2 and UB3LYP calculations on the singlet - triplet energy differences in diradicals $\mathbf{2 c}, \mathbf{1 2 b}$, and $\mathbf{1 5}$ are summarized in Table 1 . For each diradical, CASPT2 and UB3LYP give similar values of $\Delta E_{\mathrm{ST}}$, although the CASPT2 calculations uniformly give slightly higher values than UB3LYP. The values of $\Delta E_{\mathrm{ST}}$ decrease with increasing ring size. $\Delta E_{\mathrm{ST}}$ in 2c is more than five times larger than $\Delta E_{\mathrm{ST}}$ in $\mathbf{1 5}$ and more than a factor of 10 larger than $\Delta E_{\mathrm{ST}}$ in $\mathbf{1 2 \mathbf { b }}$. Clearly, the fourmembered ring in $\mathbf{2 c}$ allows much stronger through-bond interactions between the localized allylic radicals than either the five-membered ring in $\mathbf{1 5}$ or the six-membered ring in $\mathbf{1 2 b}$.

The $\left\langle S^{2}\right\rangle$ values for the "singlet" UB3LYP wave functions in Table 1 tell the same story as the $\Delta E_{\mathrm{ST}}$ values. The singlet state of hydrocarbon $\mathbf{2 c}$ has $S^{2}=0$; so by this criterion, $\mathbf{2 c}$ actually has a closed-shell B3LYP wave function. In contrast, singlet 12b and singlet 15 both have $\left\langle S^{2}\right\rangle$ values close to 1.00; so they are both predicted to have a great deal more diradical character than $2 \mathrm{c}$.

The slightly smaller value of $\left\langle S^{2}\right\rangle=0.85$ in singlet $\mathbf{1 5}$, compared with $\left\langle S^{2}\right\rangle=0.96$ in singlet $\mathbf{1 2 b}$, provides confirmatory evidence that the five-membered ring in the former diradical provides stronger through-bond interactions between the localized allylic radicals than the six-membered ring in the latter. The ratios of the NOON values, 4.3 in $\mathbf{2 c}, 1.9$ in $\mathbf{1 5}$, and 1.5 in $\mathbf{1 2 b}$, also indicate that the through-bond interactions between

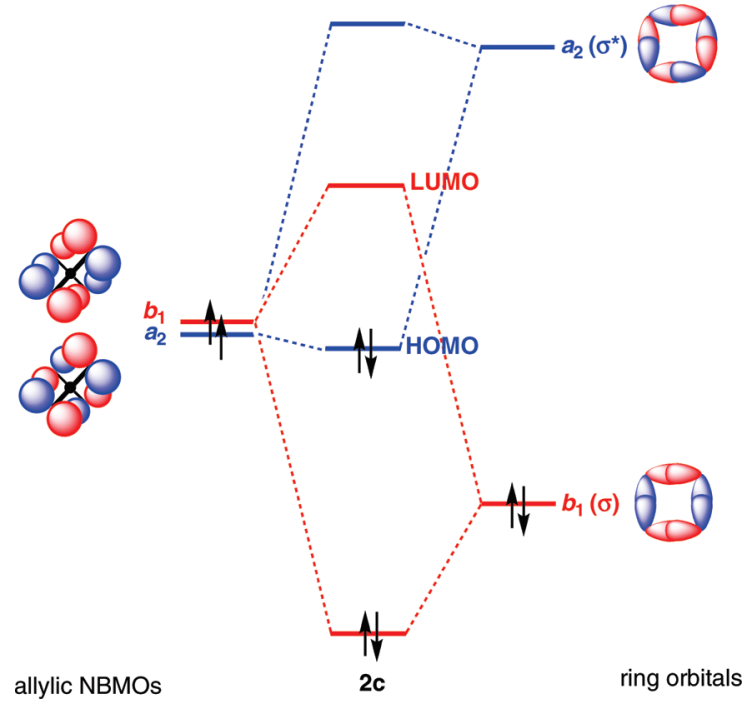

Figure 5. Orbital interaction diagram showing schematically how the $b_{1}$ and $a_{2}$ combinations of the allylic nonbonding MOs interact with the $b_{1}$ bonding and $a_{2}$ antibonding MOs of the bridged cyclobutane ring of $2 \mathrm{c}$ to create the HOMO and LUMO of the singlet state. The positions of the nodal carbons in the allylic nonbonding MOs are indicated by a dot.

the pairs of allylic radicals in these three molecules decrease as the size of the ring through which two radicals interact increases.

All of these indicators are consistent with the energy difference between the highest occupied (HO)MO and the lowest unoccupied (LU)MO in the singlet state being largest in $\mathbf{2 c}$ and smallest in $\mathbf{1 2 b}$. As shown in Table 1 the IEs of these two, singly occupied MOs in the triplet state confirm this conclusion. The UB3LYP (CASPT2) differences between the IEs of these two orbitals decrease from 43.1 (48.8) $\mathrm{kcal} / \mathrm{mol}$ in $\mathbf{2 c}$, to 17.8 (20.2) $\mathrm{kcal} / \mathrm{mol}$ in $\mathbf{1 5}$, to 13.7 (16.0) $\mathrm{kcal} / \mathrm{mol}$ in $\mathbf{1 2 b}$.

The IE values in Table 1 show that there are two contributors to the much larger energy difference between the HOMO and LUMO in $\mathbf{2 c}$ than in $\mathbf{1 5}$ and in $\mathbf{1 2 b}$. First, the four-membered ring in $2 \mathbf{c}$ interacts with the two bridging allylic radicals in such a way that the LUMO of singlet 2c (i.e., the singly occupied $\mathrm{MO}$ in the triplet with the lower IE) is about $10 \mathrm{kcal} / \mathrm{mol}$ higher in energy than the LUMOs of $\mathbf{1 5}$ and $\mathbf{1 2 b}$. However, the second and larger contributor to the much bigger HOMO-LUMO energy difference in $\mathbf{2 c}$ is the fact that the HOMO of singlet $\mathbf{2 c}$ (i.e., the singly occupied MO in the triplet with the higher IE) is ca. $20 \mathrm{kcal} / \mathrm{mol}$ lower in energy than the HOMOs of $\mathbf{1 5}$ and 12b. In order to explain these differences between the frontier orbitals of 2c and those of 15 and 12b, we begin by briefly recapitulating the previously published discussion of the interactions between the nonbonding orbitals of the two allylic radicals and the four-membered ring in $\mathbf{2 c}$. $^{3}$

As shown in Figure 5, the $b_{1}$ combination of allylic nonbonding orbitals in $\mathbf{2 c}$ interacts with only a filled, $\sigma$, bonding orbital of the four-membered ring, whereas the $a_{2}$ combination of allylic nonbonding orbitals interacts with only an unfilled, $\sigma^{*}$, antibonding orbital. The former interaction destabilizes the $b_{1}$ (in-phase) combination of allylic nonbonding orbitals; but the latter actually provides some stabilization for the $a_{2}$ (outof-phase) combination.

The resulting $a_{2}$ and $b_{1}$ MOs, which are, respectively, the HOMO and LUMO of singlet 2c, are shown in Figure 6. The energy difference between the $b_{1}$ combination of allylic nonbonding orbitals and the filled $b_{1}$ bonding orbital of the ring is considerably smaller than the energy difference between the $a_{2}$ 


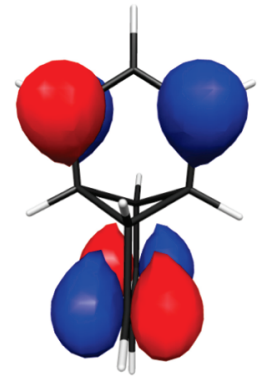

$a_{2}$

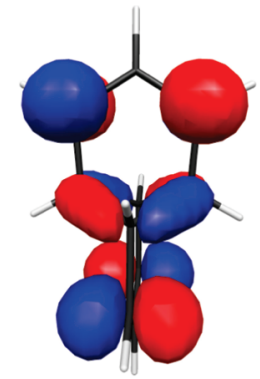

$b_{1}$
Figure 6. The $a_{2}$ HOMO and $b_{1}$ LUMO of $2 \mathrm{c}$ from B3LYP/6-31G(d) calculations. The small bonding contributions from the AOs of the ring to the $a_{2} \mathrm{MO}$ can be discerned in the teardrop shapes of the AOs at the bridging allylic carbons.

combination of allylic nonbonding orbitals and the unfilled $a_{2}$ antibonding orbital of the four-membered ring. Therefore, as shown in Figure 6, the $b_{1}$ orbital of the four-membered ring contributes much more to the LUMO of singlet $2 \mathrm{c}$ than the $a_{2}$ ring orbital contributes to the HOMO. Consequently, as illustrated schematically in Figure 5, interactions between the allylic nonbonding orbitals and the orbitals of the fourmembered ring result in more destabilization of the LUMO of the singlet state than stabilization of the HOMO.

On excitation of an electron from the HOMO to the LUMO of $\mathbf{2 c}$ to form the triplet state, an electron is removed from a MO that is weakly bonding between the four-membered ring and the unsaturated bridges and added to a MO that is strongly antibonding between the ring carbons and the bridge carbons to which the ring carbons are attached. Consequently, the lengths of the bonds between the ring and bridge carbons increase by $0.064 \AA$ on going from the singlet to the triplet state.

Why do interactions of the allylic nonbonding orbitals with the orbitals of the bridged ring create much higher energy HOMOs and much lower energy LUMOs in $\mathbf{1 5}$ and 12b than in $\mathbf{2 c}$ ? Because $\mathbf{1 2 b}$ has higher symmetry $\left(D_{2}\right)$ than $\mathbf{1 5}\left(C_{2}\right)$, we begin answering this question by comparing the orbitals of the six-membered ring in $\mathbf{1 2 b}$ with those of the four-membered ring in $2 c$.

As already noted, in the four-membered ring of $\mathbf{2 c}$ the only filled ring orbital that can interact with either symmetry combination of the allylic nonbonding MOs is $b_{1}$. In contrast, in the six-membered ring of $\mathbf{1 2 b}$ there is not only a filled ring orbital of symmetry $a$ in the $D_{2}$ point group that can interact with the in-phase combination of allylic nonbonding MOs but also a filled ring orbital of symmetry $b_{1}$ that can interact with the out-of-phase combination. These $a$ and $b_{1}$ orbitals of the six-membered ring in $\mathbf{1 2 b}$ are shown schematically in the center of Figure $7 .^{16,17}$

Figure 7 depicts the relationship between the $a$ and $b_{1}$ orbitals of the six-membered ring in $\mathbf{1 2 b}$ and the corresponding orbitals of the four- and five-membered rings in, respectively, $\mathbf{2 c}$ and 15. As shown in Figure 7, the filled orbital of the six-membered ring in $12 \mathrm{~b}$ that belongs to the $a$ representation of $D_{2}$ bears a

(16) For consistency, the orbital lobes in Figure 7 have all been drawn so that they lie along the $\mathrm{C}-\mathrm{C}$ bonds of the six-membered ring, but the actual AOs do not lie along the bonds. The orbital lobes behave like vectors, so, for instance, the AOs at C-3 and C-6 in the $a$ MO are actually hybrid orbitals that point toward the center of the sixmembered ring, whereas in the $b_{1} \mathrm{MO}$ the AOs at these two carbons are pure $2 \mathrm{p}$ AOs that are tangential to the ring. strong resemblance to the filled $b_{1}$ orbital of the four-membered ring in 2c. In fact, as indicated in Figure 7, upon excision of the two equivalent carbons, C-3 and C-6, from $\mathbf{1 2 b}$ and attachment of C-1 to C-5 and C-2 to C-4, the $a$ orbital of $\mathbf{1 2 b}$ is transformed into the $b_{1}$ orbital of $\mathbf{2 c}$.

Figure 7 also shows how, in similar fashion, the $b_{1}$ ring orbital of $\mathbf{1 2} \mathbf{b}$ is related to the $a_{2}$ ring orbital of $\mathbf{2 c}$. The $b_{1}$ ring orbital of 12b is bonding between C-3 and both C-2 and C-4 and between $\mathrm{C}-6$ and both $\mathrm{C}-1$ and $\mathrm{C}-5$. Although this ring orbital is antibonding between $\mathrm{C}-1$ and $\mathrm{C}-2$ and between $\mathrm{C}-4$ and $\mathrm{C}-5$, the AOs at C- 1 and $\mathrm{C}-2$ and at $\mathrm{C}-4$ and $\mathrm{C}-5$ are hybridized away from each other, so their antibonding interaction is minimized. Consequently, the $b_{1}$ orbital of the six-membered ring in $\mathbf{1 2 b}$ is a doubly occupied bonding orbital.

On the other hand, if C-3 and C- 6 of the six-membered ring are excised and C- 1 and C-5 and C-2 and C- 4 are attached to each other, the $b_{1}$ ring orbital of $\mathbf{1 2 b}$ becomes the $a_{2}$ ring orbital of 2c. Since the $a_{2}$ orbital has nodes between every pair of carbons in the four-membered ring of $\mathbf{2 c}, a_{2}$ is an antibonding orbital. Consequently, as shown schematically in Figure 5, this ring orbital is unoccupied in $\mathbf{2} \mathbf{c}$ and higher in energy than the $a_{2}$ combination of allylic nonbonding $\pi$ MOs.

In summary, the right-hand side of Figure 7 reveals why the $b_{1}$ orbital of the six-membered ring in $\mathbf{1 2} \mathbf{b}$ is bonding and occupied but the $a_{2}$ orbital of the four-membered ring in $2 \mathbf{c}$ is antibonding and empty. This is the most important difference between the orbitals of the six-membered ring in $\mathbf{1 2 b}$ and those of the four-membered ring in $\mathbf{2 c}$.

As shown schematically in Figure 8 , the $b_{1}$ orbital of the sixmembered ring in $\mathbf{1 2 b}$ destabilizes the out-of-phase combination of allylic nonbonding $\pi$ MOs, rather than stabilizing this combination, as the $a_{2}$ ring orbital does in $2 \mathbf{c}$ (Figure 5). This difference between the orbital interactions in $\mathbf{1 2 b}$ and $\mathbf{2 c}$ is responsible for the ca. $20 \mathrm{kcal} / \mathrm{mol}$ difference between the energies (Table 1) of the MOs that are the HOMOs of the singlet states of these two molecules.

Figure 7 reveals that there is also a significant difference between the orbital of the six-membered ring in $\mathbf{1 2 b}$ that belongs to the $a$ representation of $D_{2}$ and the orbital of the fourmembered ring of $\mathbf{2 c}$ that belongs to the $b_{1}$ representation of $D_{2 d}$. The former orbital has a large contribution from the two unique carbons of the six-membered ring in $\mathbf{1 2 b}$; but these carbons do not interact with the bridging allylic groups. Consequently, the $a$ orbital of the six-membered ring in $\mathbf{1 2 b}$ is less effective than the $b_{1}$ ring orbital of the four-membered ring in $\mathbf{2} \mathbf{c}$ at destabilizing the LUMO by mixing with the in-phase

(17) The MOs are accurately depicted in Jorgensen, W. L.; Salem, L. The Organic Chemist's Book of Orbitals; Academic Press: New York, 1973. The MOs of cyclobutane have been discussed by Salem, L.; Wright, J. S. J. Am. Chem. Soc. 1969, 91, 3947. And by Hoffmann, R.; Davidson, R. B. J. Am. Chem. Soc. 1971, 93, 5699. The MOs of six-membered rings have been discussed by Hoffmann, R.; Molère, P. D.; Heilbronner, E. J. Am. Chem. Soc. 1973, 95, 4860.

(18) Another possible explanation for the higher LUMO energy of $\mathbf{2} \mathbf{c}$ than of 12b might be that the $b_{1}$ ring orbital of the four-membered ring in 2c is higher in energy than the $a$ ring orbital of the six-membered ring of 12b. Were this the case, then the closer energetic proximity between the $b_{1}$ ring orbital and the in-phase combination of allylic nonbonding orbitals would explain why the $b_{1}$ cyclobutane ring orbital destabilizes the LUMO of $\mathbf{2 c}$ more than the $a$ cyclohexane ring orbital destabilizes the LUMO of $\mathbf{1 2} \mathbf{b}$. However, as shown in Table 2, the $b_{1}$ orbital of the four-membered ring is actually $30-40 \mathrm{kcal} / \mathrm{mol}$ lower in energy than the $a$ orbital of the six-membered ring. Consequently, it must be the weaker interaction of the in-phase combination of allylic nonbonding MOs with the $a$ ring orbital of $\mathbf{1 2} \mathbf{b}$ than with the $b_{1}$ ring orbital of $\mathbf{2 c}$ that is responsible for the $10-12 \mathrm{kcal} / \mathrm{mol}$ lower energy of the LUMO of $\mathbf{1 2 b}$ than of $\mathbf{2 c}$. 

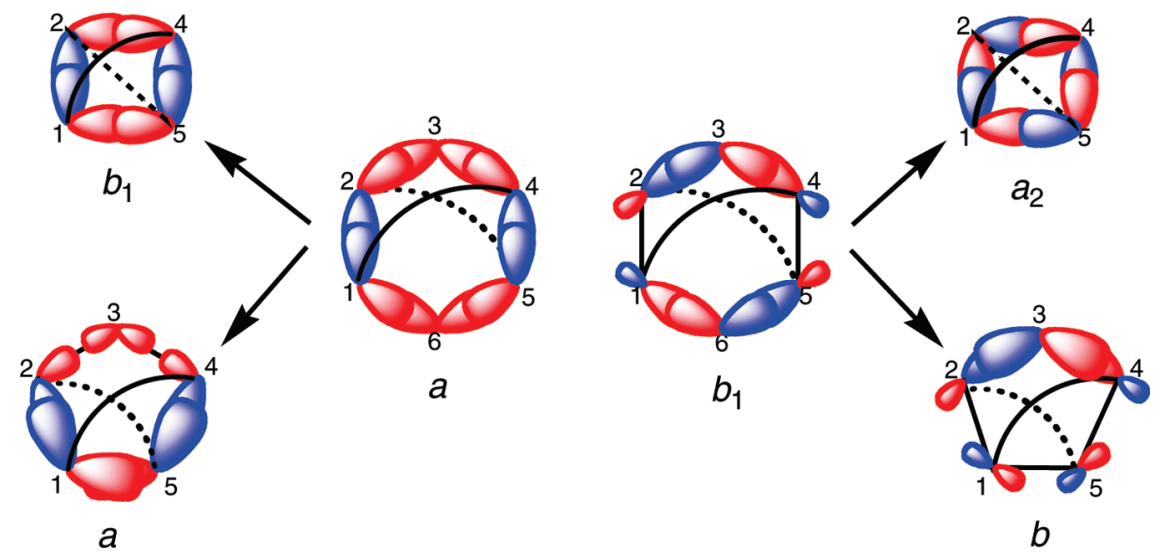

Figure 7. Schematic depiction of the nodal patterns in the bonding $a$ and $b_{1}$ orbitals of the six-membered ring in $\mathbf{1 2 b}$ and of how, by excision of one or two carbons, these orbitals can be related to, respectively, the $a$ and $b$ ring orbitals of the five-membered ring of $\mathbf{1 5}$ or to the $b_{1}$ and $a_{2}$ orbitals of the four-membered ring in $\mathbf{2 c}$. The allylic groups that bridge the rings on opposite sides of $\mathbf{2 c}, \mathbf{1 5}$, and $\mathbf{1 2 b}$ are represented by the curved lines that connect $\mathrm{C}-1$ with C-4 and C-2 with C-5.
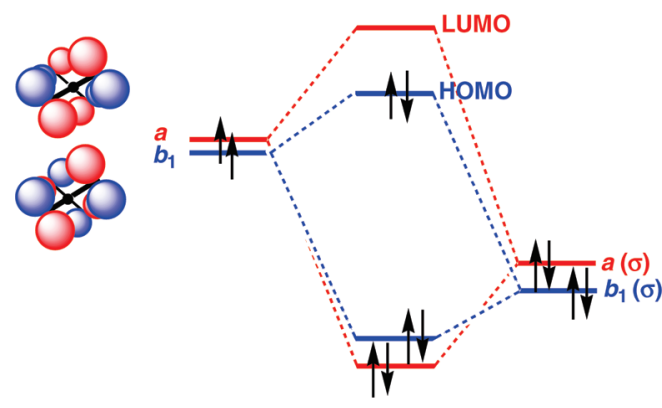

allylic NBMOs

$12 b$

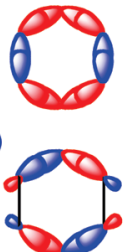

ring orbitals

Figure 8. Interaction diagram showing schematically how the $a$ and $b_{1}$ combinations of the allylic nonbonding MOs interact with the $a$ and $b_{1}$ bonding MOs of the bridged cyclohexane ring to create the HOMO and LUMO of singlet diradical $\mathbf{1 2 b}$. The positions of the nodal carbons in the allylic nonbonding MOs are indicated by a dot.

combination of the allylic nonbonding MOs. Indeed, we attribute the $10-12 \mathrm{kcal} / \mathrm{mol}$ higher energy of the LUMO in $2 \mathrm{c}$ than in 12b (Table 1) to the weaker interaction of the in-phase combination of allylic nonbonding MOs with the $a$ ring orbital of $\mathbf{1 2} \mathbf{b}$ than with the $b_{1}$ ring orbital of $\mathbf{2} \mathbf{c} .^{18}$

Comparison of Figure 8 with Figure 5 illustrates graphically the two reasons, discussed above, for the ca. $30 \mathrm{kcal} / \mathrm{mol}$ larger HOMO-LUMO energy gap in $\mathbf{2 c}$ than in $\mathbf{1 2 b}$. About $20 \mathrm{kcal} /$ mol of the larger energy gap in $\mathbf{2 c}$ comes from the presence of the filled $b_{1}$ orbital in the six-membered ring, which destabilizes the out-of-phase combination of allylic nonbonding MOs in the HOMO of $\mathbf{1 2 b}$. Another ca. $10 \mathrm{kcal} / \mathrm{mol}$ comes from the weaker interaction of the $a$ orbital of the six-membered ring, compared with the $b_{1}$ orbital of the four-membered ring, with the in-phase combination of allylic nonbonding MOs in the LUMO. It is, of course, the much larger HOMO-LUMO energy gap in 2c than in $\mathbf{1 2 b}$ that leads to $\Delta E_{\mathrm{ST}}$ being computed to be $20-22 \mathrm{kcal} /$ mol larger in $\mathbf{2 c}$ than in $\mathbf{1 2 b}$.

Unlike the case in $\mathbf{2 c}$, where formation of the triplet from the singlet requires excitation of an electron from an MO that is weakly bonding between the ring and the bridge into an MO that is strongly antibonding, Figure 8 indicates that in $\mathbf{1 2 b}$ both the HOMO and LUMO are antibonding between the ring and the bridge. Consequently, forming the triplet from the singlet should result in a smaller increase in the length of the bonds between the ring and bridge in $\mathbf{1 2 b}$ than in $\mathbf{2 c}$.
Table 2. Energies $(\mathrm{kcal} / \mathrm{mol})$ of the Filled Ring Orbitals ${ }^{a}$ That Interact with the In-Phase and Out-of-Phase Combination of Allylic Nonbonding Orbitals in 2c, 15, and 12b

\begin{tabular}{cccccc}
\hline $\begin{array}{c}\text { type of } \\
\text { MO energy }\end{array}$ & $\begin{array}{c}b_{1} \text { MO } \\
\text { of 2c }\end{array}$ & $\begin{array}{c}\text { a MO } \\
\text { of } 15\end{array}$ & $\begin{array}{c}b \text { MO } \\
\text { of } 15\end{array}$ & $\begin{array}{c}\text { a MO } \\
\text { of 12b }\end{array}$ & $\begin{array}{c}b_{1} \text { MO } \\
\text { of 12b }\end{array}$ \\
\hline HF orbital & -286.5 & -275.6 & -281.5 & -259.1 & -264.6 \\
-(U)B3LYP IE & -262.4 & -246.9 & $b$ & -227.5 & -231.4
\end{tabular}

${ }^{a}$ Calculated for the $\mathrm{C}_{n} \mathrm{H}_{2 n}$ ring at the optimized geometry of the doubly bridged tricyclic molecule but with the $\mathrm{C}-\mathrm{C}$ bonds to the bridges replaced by $\mathrm{C}-\mathrm{H}$ bonds, whose lengths were optimized. ${ }^{b}$ This ring orbital is not the highest of $b$ symmetry in $\mathbf{1 5}$, so an attempt to compute the IE of this orbital leads, instead, to the IE of the lower energy $b$ orbital.

Unfortunately, the UB3LYP wave function for singlet $\mathbf{1 2 b}$ has $\left\langle S^{2}\right\rangle=0.96$. Therefore, the $1.505 \AA$ length of the bonds between the ring and bridge in the UB3LYP optimized geometry of "singlet" $\mathbf{1 2 b}$ is probably close to a $1: 1$ average of this bond length in the triplet $(1.515 \AA)$ and this bond length in the pure $\left(S^{2}=0.0\right)$ singlet. It can then be inferred that the bonds between the ring and the bridge are $1.495 \AA$ long in the pure singlet state of $\mathbf{1 2 b}$. If this inference is correct, the length of these bonds increases by $0.020 \AA$ on going from the pure singlet to the triplet state of $\mathbf{1 2 b}$. As expected, this increase is much smaller than the $0.064 \AA$ increase in the length of these bonds between the ring and bridge on going from the singlet to the triplet state of 2c.

Figure 8 raises the question, why does the filled $a$ orbital of the six-membered ring in $\mathbf{1 2 b}$ interact more strongly with the in-phase combination of allylic nonbonding orbitals than the filled $b_{1}$ ring orbital interacts with the out-of phase combination? Table 2, which gives the energies of the highest-lying filled MOs of the saturated rings in diradicals $\mathbf{2 c}, \mathbf{1 2 b}$, and $\mathbf{1 5}$, shows that the $a$ orbital of the six-membered ring in $\mathbf{1 2 b}$ is higher in energy than the $b_{1}$ ring orbital by $4-6 \mathrm{kcal} / \mathrm{mol}$. However, this small energy difference between these ring orbitals cannot explain why the $a$ LUMO of 12b lies $14-16 \mathrm{kcal} / \mathrm{mol}$ higher in energy than the $b_{1}$ HOMO.

Inspection of Figure 7 reveals why, in $\mathbf{1 2 b}$, the $a$ orbital of the six-membered ring interacts more strongly with the in-phase combination of allylic MOs than the $b_{1}$ ring orbital interacts with the out-of-phase combination. The $a$ ring orbital has larger coefficients than the $b_{1}$ ring orbital at C-1, C-2, C-4, and C-5, the four carbons to which the two allyl bridging groups are attached. It is easy to understand why this should be the case, 


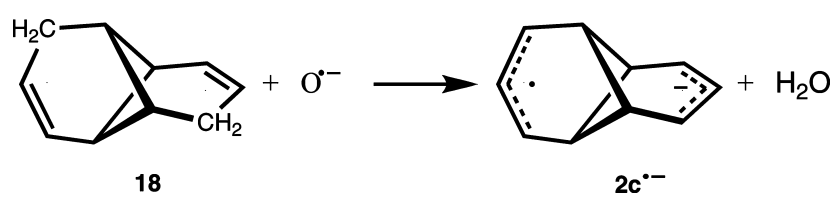

Figure 9. A possible reaction for generating radical anion $2 \mathrm{c}^{\mathbf{*}}$ in the gas phase for negative ion photoelectron spectroscopy (NIPES), in order to measure $\Delta E_{\mathrm{ST}}$ in $\mathbf{2 c}$.

because the interaction of $\mathrm{C}-1$ with $\mathrm{C}-2$ and $\mathrm{C}-4$ with $\mathrm{C}-5$ is bonding in the $a$ ring orbital but antibonding in the $b_{1}$ ring orbital. In order to minimize the effect of these antibonding interactions on the energy of the $b_{1}$ ring orbital, the coefficients at C-1, C-2, C-4, and C-5 are smaller in the $b_{1}$ orbital than in the $a$ orbital of the six-membered ring of $\mathbf{1 2 b}$.

The same type of consideration of the coefficients at C- 1 and C-5 in the $a$ and $b$ orbitals of the five-membered ring in $\mathbf{1 5}$ (Figure 7) leads to the expectation that the in-phase combination of allylic orbitals should be destabilized by its interaction with the $a$ ring orbital by more than the out-of-phase combination of allylic orbitals should be destabilized by its interaction with the $b$ ring orbital. Table 1 shows that, in fact, the $18-20 \mathrm{kcal} /$ mol difference between the energies of the $a$ and $b$ MOs of 15 is about $4 \mathrm{kcal} / \mathrm{mol}$ larger than the difference between the $a$ and $b_{1}$ MOs of $\mathbf{1 2} \mathbf{b}$. This slightly larger HOMO-LUMO energy difference in $\mathbf{1 5}$ than in $\mathbf{1 2 b}$ is responsible for the fact that, as shown in Table $1, \Delta E_{\mathrm{ST}}$ is $2.1-3.5 \mathrm{kcal} / \mathrm{mol}$ larger for $\mathbf{1 5}$ than for $\mathbf{1 2 b}$.

\section{Summary and Conclusions}

Unlike the case in $\mathbf{1 5}$ or in $\mathbf{1 2 b}$, the HOMO of $\mathbf{2 c}$ is not destabilized by the interaction of the $a_{2}$ combination of allylic orbitals with a filled ring orbital. In fact, interaction with an unfilled $a_{2}$ ring orbital actually stabilizes the HOMO of $\mathbf{2 c}$, which helps to make the HOMO of 2c 17-18 kcal/mol lower in energy than the HOMO of 15 and $19-20 \mathrm{kcal} / \mathrm{mol}$ lower than the HOMO of $\mathbf{1 2 b}$. In addition, the filled $b_{1}$ ring orbital of 2c interacts more strongly with the in-phase combination of allylic nonbonding orbitals than the filled $a$ ring orbital of both 15 and $\mathbf{1 2 b}$. Consequently, the $b_{1}$ LUMO of $\mathbf{1 2 b}$ is higher in energy by $9-10 \mathrm{kcal} / \mathrm{mol}$ than the LUMO of $\mathbf{1 5}$ and by $10-12$ $\mathrm{kcal} / \mathrm{mol}$ than the LUMO of $\mathbf{1 2 b}$. Thus, the HOMO-LUMO energy gap in $\mathbf{2 c}$ is $26-28 \mathrm{kcal} / \mathrm{mol}$ larger than that in $\mathbf{1 5}$ and 29-32 kcal/mol larger than that in $\mathbf{1 2 b}$. The larger HOMOLUMO energy difference in $2 \mathbf{c}$ makes $\Delta E_{\mathrm{ST}}$ in $\mathbf{2 c} 18-20 \mathrm{kcal} /$ mol larger than in $\mathbf{1 5}$ and $20-23 \mathrm{kcal} / \mathrm{mol}$ larger than in $\mathbf{1 2 b}$.

The prediction of a very large value of $\Delta E_{\mathrm{ST}}$ in $2 \mathbf{c}$ should be, at least in principle, testable experimentally. As shown in Figure 9, generation of the radical anion of $\mathbf{2 c}$ should be possible by reaction of diene $\mathbf{1 8}$ with $\mathrm{O}^{\circ-}$. (U)B3LYP/6-31+G(d) calculations predict that the odd electron in $\mathbf{2 \mathbf { c } ^ { - }}$ is bound by $28.9 \mathrm{kcal} / \mathrm{mol}$, so negative ion photoelectron spectroscopy (NIPES) of $\mathbf{2} \mathbf{c}^{--}$should allow $\Delta E_{\mathrm{ST}}$ in $\mathbf{2 c}$ to be measured. ${ }^{19}$

(19) Similar $\mathrm{O}^{--}$reactions with the appropriate hydrocarbon allowed the generation of the radical anion of tetramethyleneethane: Lee, J.; Chou, P. K.; Dowd, P.; Grabowski, J. J. J. Am. Chem. Soc. 1993, 115, 7902. NIPES on the radical anion was then used to measure $\Delta E_{\mathrm{ST}}$ in TME: Clifford, E. P.; Wenthold, P. G.; Lineberger, W. C.; Ellison, G. B.; Wang, C. X.; Grabowski, J. J.; Vila, F.; Jordan, K. D. J. Chem. Soc., Perkins Trans. 2 1998, 1015. The same chemistry was used to generate the radical anion of MBQDM, so that the value of $\Delta E_{\mathrm{ST}}$ could be measured by NIPES: Wenthold, P. G.; Kim, J. B.; Lineberger, W. C. J. Am. Chem. Soc. 1997, 119, 1354.
The large value of $\Delta E_{\mathrm{ST}}$ in $2 \mathrm{c}$ should also manifest itself in the thermodynamics for formation of singlet $\mathbf{2 c}$ by breaking two $\mathrm{C}-\mathrm{H}$ bonds in 18. Our CASPT2 calculations find that breaking the first $\mathrm{C}-\mathrm{H}$ bond in $\mathbf{1 8}$ to give radical 19 requires $3.4 \mathrm{kcal} / \mathrm{mol}$ less energy than breaking the second $\mathrm{C}-\mathrm{H}$ bond to give triplet 2c (Figure 10). Perhaps not surprisingly, the fourmembered ring provides more hyperconjugative stabilization for the single allylic radical in $\mathbf{1 9}$ than for the pair of allylic radicals in triplet $\mathbf{2 c}$.

If the heat of formation of triplet $\mathbf{2 c}$ could be accurately predicted from the heat of formation of $\mathbf{1 8}$ plus twice the $\mathrm{C}-\mathrm{H}$ BDE for forming 19 from 18, the heat of formation of triplet $2 \mathrm{c}$ would be said to follow bond enthalpy additivity (BEA). ${ }^{20}$ However, as already noted, the heat of formation of triplet $\mathbf{2 c}$ is expected to show a positive deviation from BEA, because CASPT2 predicts that in forming triplet $2 \mathrm{c}$ from $\mathbf{1 8}$, a $\mathrm{C}-\mathrm{H}$ bond in $\mathbf{1 9}$ is harder to break than a $\mathrm{C}-\mathrm{H}$ bond in $\mathbf{1 8}$ by $\triangle \mathrm{BDE}$ $=3.4 \mathrm{kcal} / \mathrm{mol}$.

The CASPT2 value of $\Delta E_{\mathrm{ST}}=25.5 \mathrm{kcal} / \mathrm{mol}$ in $2 \mathrm{c}$ means that breaking the second $\mathrm{C}-\mathrm{H}$ bond in $\mathbf{1 9}$ to form the singlet state of $\mathbf{2 c}$ should require this much less energy than forming the triplet. Therefore, breaking the second $\mathrm{C}-\mathrm{H}$ bond in $\mathbf{1 9}$ to form singlet $2 \mathrm{c}$ should be easier than breaking the first $\mathrm{C}-\mathrm{H}$ bond in 18 to form 19 by $3.4-25.5=-22.1 \mathrm{kcal} / \mathrm{mol}$. This is the predicted size of the negative deviation from BEA in forming singlet $2 \mathrm{c}$ by breaking two $\mathrm{C}-\mathrm{H}$ bonds in $\mathbf{1 8}$.

The large negative deviation from BEA that is predicted for forming singlet $\mathbf{2 c}$ from $\mathbf{1 8}$ is a consequence of the fact that in contrast to the case in forming triplet $2 \mathbf{c}$, both of the nonbonding electrons in singlet 2c can occupy an MO $\left(a_{2}\right)$ that is not destabilized by interactions with any of the filled MOs of the four-membered ring. In fact, the HOMO of singlet $\mathbf{2 c}$ is actually stabilized by the bonding interactions between the out-of-phase combination of allyl nonbonding orbitals and the unfilled $a_{2}$ orbital of the doubly bridged four-membered ring. Thus, for the same reason that a large value of $\Delta E_{\mathrm{ST}}$ is predicted for $\mathbf{2 c}$, the singlet state of $\mathbf{2 c}$ is predicted to show a very large negative deviation from BEA. ${ }^{21}$

The large thermodynamic stabilization of singlet $2 \mathbf{c}$ by through-bond interactions is also responsible for the surprisingly small enthalpy difference that is computed between this singlet diradical and bicyclic tetraene 1c. Despite the fact that diradical $\mathbf{2 c}$ contains a four-membered ring, which is absent in $\mathbf{1 c}$, the enthalpy difference between 2c and 1c amounts to only 25.6 $\mathrm{kcal} / \mathrm{mol}$. One might have imagined that the strain in the fourmembered ring of $\mathbf{2 c}$ would have made the enthalpy difference

(20) For a review and critical discussion of deviations from BEA, see: Zhang, D. Y.; Borden, W. T. J. Org. Chem. 2002, 67, 3989.

(21) On replacing the two allyl bridging groups in $\mathbf{2}$ with a pair of double bonds, the symmetries of the frontier orbitals of the bridges change from $b_{1}$ and $a_{2}$ in 2 to $e$ in bicyclo[3.3.0.0 $0^{3,7}$ ]octa-2,6-diene. This means that the latter molecule is destabilized, because both of the occupied $\pi$ orbitals of the ethylenic bridges interact with a filled orbital of the cyclobutane ring. ${ }^{22}$ Phenomena as diverse as the rapid rearrangement of bicyclo[3.3.0.0 ${ }^{3,7}$ octa-2,6-diene to semibullvalene at $25^{\circ} \mathrm{C},{ }^{23 a, b}$ the very high heat of hydrogenation of the $\pi$ bond in bicyclo[2.1.1] hex-2-ene, ${ }^{23 \mathrm{c}, \mathrm{d}}$ and the manner in which annelation by 1,3-bridged cyclobutane rings causes the double bonds in benzene, ${ }^{23 \mathrm{e}-\mathrm{g}}$ cyclooctatetraene, ${ }^{23 \mathrm{~h}, \mathrm{i}}$ and naphthalene $e^{23 j}$ to localize can all be attributed to this type of destabilizing interaction between the filled $\pi$ orbital of ethylene and a filled orbital of a cyclobutane ring that is 1,3-bridged by a double bond. The long wavelength UV/vis absorptions seen in molecules containing a cyclobutane ring that is 1,3 -bridged by ethylene ${ }^{24 a-d}$ have been attributed to the same type of interaction between occupied orbitals of the ring and of the unsaturated bridge. ${ }^{24 \mathrm{e}-\mathrm{g}}$ 


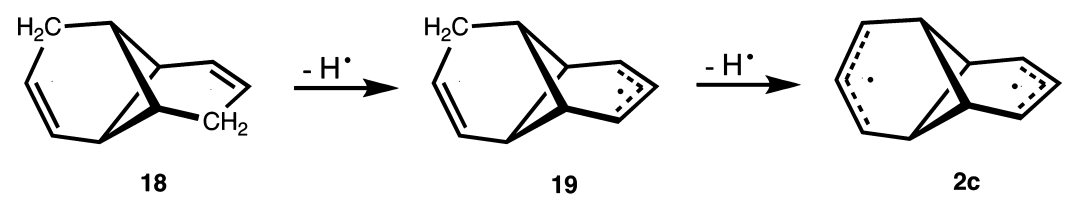

Figure 10. Reactions involved in testing whether the heat of formation of $\mathbf{2 c}$ deviates from bond enthalpy additivity (BEA). BEA involves the naïve assumption that the BDEs of an allylic $\mathrm{C}-\mathrm{H}$ bond in $\mathbf{1 8}$ and $\mathbf{1 9}$ are the same.

between $\mathbf{2 c}$ and $\mathbf{1 c}$ much larger than the $23.9 \mathrm{kcal} / \mathrm{mol}$ enthalpy difference between $\mathbf{1 2 b}$ and $\mathbf{1 1 b}$.

Actually the difference in strain energy between the fourmembered ring, created in the cyclization of $\mathbf{1 c}$ to $\mathbf{2 c}$, and the six-membered ring, created in the cyclization of $\mathbf{1 1 b}$ to $\mathbf{1 2 b}$, does manifest itself clearly in the difference between the enthalpies of these two cyclization reactions. However, the difference in strain energies manifests itself in the difference between the enthalpies of the cyclization reactions of $\mathbf{1 c}$ and 11b to form the triplet, not the singlet, diradicals.

The difference between the enthalpies of cyclization of 1c and $\mathbf{1 1 b}$ to form, respectively, triplet diradicals $2 \mathrm{c}$ and $\mathbf{1 2 c}$ can easily be obtained by combining the ca. $2 \mathrm{kcal} / \mathrm{mol}$ difference between the enthalpies of cyclization of $\mathbf{1 c}$ and $\mathbf{1 1 b}$ in forming

(22) Jorgensen, W. L.; Borden, W. T. J. Am. Chem. Soc. 1973, 95, 6649. (23) (a) Meinwald, J.; Schmidt, D.; Tsruta, J. Am. Chem. Soc. 1969, 91, 5877. (b) Zimmerman, H. E.; Robbins, J. D.; Schantl, J. J. Am. Chem. Soc. 1969, 91, 5878. (c) Wiberg, K. B.; Bonneville, G.; Dempsey, R. Isr. J. Chem. 1983, 23, 85. (d) Wiberg, K. B.; Wasserman, D. J.; Martin, E. J.; Murcko, M. A. J. Am. Chem. Soc. 1985, 107, 6019. (e) Baldridge, K. K.; Siegel, J. S. J. Am. Chem. Soc. 1992, 114, 9583. (f) Frank, N. L.; Baldridge, K. K.; Siegel, J. S. J. Am. Chem. Soc. 1995, 117, 2102. (g) Burgi, H.-B.; Baldridge, K. K.; Hardcastle, K.; Frank, N. L.; Ganzel, P.; Siegel, J. S.; Ziller., J. Angew. Chem., Int. Ed., Engl. 1995, 34, 1454. (h) Baldridge, K. K.; Siegel, J. S. J. Am. Chem. Soc. 2001, 123, 1755. (i) Matsura, A.; Komatsu, K. J. Am. Chem. Soc. 2001, 123, 1768. (j) Uto, T.; Nishinaga, T.; Matsura, A.; Inoue, R.; Komatsu, K. J. Am. Chem. Soc. 2005, 127, 10162.

(24) (a) Meinwald, J.; Kaplan, B. E. J. Am. Chem. Soc. 1967, 89, 261. (b) Meinwald, J.; Uno, F. J. Am. Chem. Soc. 1968, 90, 800. (c) Robin, M. B.; Basch, H.; Keubler, N. A.; Kaplan, B. E.; Meinwald, J. J. Chem. Phys. 1968, 48, 5037. (d) Meinwald, J.; Tsuruta, J. Am. Chem. Soc. 1970, 98, 2579. (e) Gleiter, R.; Kobayashi, T. Helv. Chem. Acta 1971, 54, 1081. (f) Hoffmann, R.; Davidson, R. B. J. Am. Chem. Soc. 1970, 98, 2579. (g) Bischof, P.; Gleiter, R.; Kukla, M. J.; Paquette, L. J. Electron Spectrosc. Relat. Phenom. 1974, 4, 177. the singlet diradicals with the 20 (UB3LYP) to 23 (CASPT2) $\mathrm{kcal} / \mathrm{mol}$ larger value of $\Delta E_{\mathrm{ST}}$ in $\mathbf{2 c}$ than in $\mathbf{1 2 b}$. The enthalpy of cyclization of $\mathbf{1 c}$ to form triplet $\mathbf{2 c}$ is thus calculated to be $22-25 \mathrm{kcal} / \mathrm{mol}$ higher than that of $\mathbf{1 1 b}$ to form triplet $\mathbf{1 2 b}$.

In forming the singlet diradicals, the $22-25 \mathrm{kcal} / \mathrm{mol}$ larger strain in the cyclization of $\mathbf{1 c}$ to triplet $\mathbf{2 c}$ compared with the cyclization of $\mathbf{1 1 b}$ to triplet $\mathbf{1 2} \mathbf{b}$ is nearly canceled by the $20-23$ $\mathrm{kcal} / \mathrm{mol}$ larger through-bond stabilization of singlet $\mathbf{2 c}$, relative to singlet $\mathbf{1 2 b}$. This near cancellation is the reason the enthalpies of cyclization of $\mathbf{1 c}$ to singlet $\mathbf{2 c}$ and $\mathbf{1 1 b}$ to singlet $\mathbf{1 2 b}$ are nearly the same, despite the formation of a strained fourmembered ring in the former reaction.

The transition structure leading from $\mathbf{1 c}$ to singlet $\mathbf{2 c}$ is computed to be only about $2 \mathrm{kcal} / \mathrm{mol}$ higher in enthalpy than 2c; so it seems highly likely that the through-bond interactions that stabilize singlet $\mathbf{2 c}$ also stabilize the transition structure leading to this singlet diradical. Consequently, the unexpectedly low barriers measured for the cyclizations of $\mathbf{1 a} \rightarrow \mathbf{2 a}$ and $\mathbf{1 b}$ $\rightarrow \mathbf{2} \mathbf{b}^{1}$ and calculated for the cyclization of $\mathbf{1 c} \rightarrow \mathbf{2 c}$ can be reasonably attributed to through-bond stabilization of the transition structures for formation of the singlet diradicals.

Acknowledgment. We thank the National Science Foundation for Grant CHE-0910527 and the Robert A. Welch Foundation for Grant B-0027 in support of this research at UNT.

Supporting Information Available: Optimized geometries and energies for the hydrocarbons, radicals, diradicals, and ions discussed in this paper and complete ref 15 . This material is available free of charge via the Internet at http://pubs.acs.org.

JA106329T 